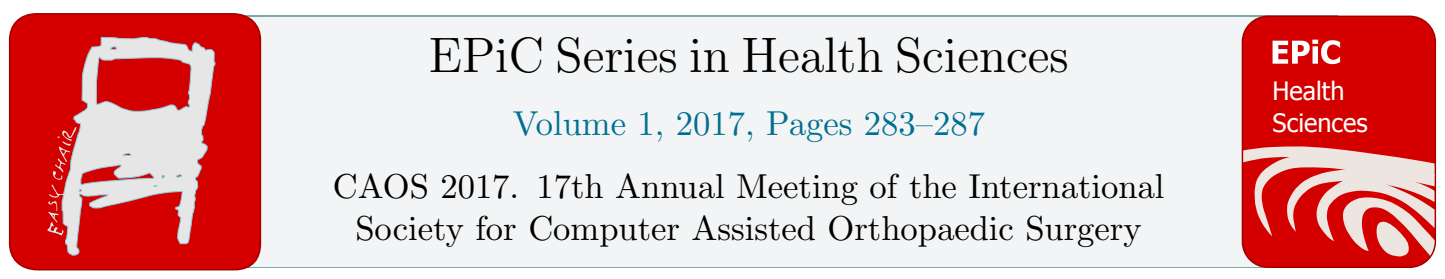

\title{
Robotic-Arm Assisted Total Knee Arthroplasty Demonstrated Greater Accuracy to Plan Compared to Manual Technique
}

\author{
Emily L. Hampp $\mathrm{PhD}^{1}$, Laura Y. Scholl MSc ${ }^{1}$, Ta-Cheng Chang $\mathrm{PhD}^{1}$, Abdullah Abbasi $\mathrm{MSc}^{1}$, \\ Manoshi Bhowmik-Stoker $\mathrm{PhD}^{1}$, Jason K. Otto $\mathrm{PhD}^{1}$, David J. Jacofsky $\mathrm{MD}^{2}$ and Michael A. Mont \\ $\mathrm{MD}^{3}$ \\ ${ }^{1}$ Stryker, Mahwah, NJ, USA \\ ${ }^{2}$ The Core Institute, Phoenix, AZ, USA \\ ${ }^{3}$ Cleveland Clinic, Cleveland, OH, USA \\ emily.hampp@stryker.com, laura.sholl@stryker.com, \\ ta.Chang@stryker.com, abdullah.abbasi@stryker.com, manoshi.bhowmik- \\ stoker@stryker.com, jason.otto@stryker.com, \\ david.jacofsky@thecoreinstitute.com, montm@ccf.org
}

\begin{abstract}
While total knee arthroplasty has demonstrated clinical success, final bone cut and final component alignment can be critical for achieving a desired overall limb alignment. This cadaver study investigated whether robotic-arm assisted total knee arthroplasty (RATKA) allows for accurate bone cuts and component position to plan, compared to manual technique. Six cadaveric specimens (12 knees) were prepared by an experienced user of manual total knee arthroplasty (MTKA), who was inexperienced in RATKA. For each cadaveric pair, a RATKA was prepared on the right leg and a MTKA was prepared on the left leg. Final bone cuts and final component position to plan were measured relative to fiducials, and medians and standard deviations were compared. Accuracy to plan was defined by the median values of the absolute deviation from plan. Standard deviation was used to describe the precision to plan. Two-Variance assessment using Levene's test was performed to evaluate for differences between precision of the two techniques.

When comparing all six matched pairs, RATKA bone cuts were as or more accurate to plan than the MTKA control for 11/12 bone cut measurements. Similarly, RATKA bone cuts were as or more precise to plan than the MTKA control on all femoral bone cuts. Additionally, RATKA final component positions were as or more accurate to plan than the MTKA control for all measurements. Similarly, RATKA final component positions were as or more precise to plan than MTKA for all femoral implant positions, as well as the tibial varus/valgus implant position. A comparison of the standard deviations for the last three RATKA and MTKA matched pairs showed that RATKA was
\end{abstract}


more precise to plan for all cuts and implant positions compared to MTKA, including tibial antero-posterior bone cut, as well as tibial implant antero-posterior slope positioning.

In general, RATKA demonstrated greater accuracy and precision of bone cuts and component placement to plan, respectively, compared to MTKA in this cadaveric study. For further confirmation, RATKA accuracy of component placement should be investigated in a clinical setting.

\section{Introduction}

While total knee arthroplasty has demonstrated clinical success [1,2], a meta-analysis of component alignment found mechanical axis malalignment of greater than $3^{\circ}$ in $9.0 \%$ of computer-assisted (CAS) and $31.8 \%$ of conventional TKA surgeries [3]. This study aimed to determine whether robotic-arm assisted total knee arthroplasty (RATKA) allows for accurate bone cuts and component position to plan, compared to manual technique. Additionally, this study investigated whether RATKA allows an experienced user of manual total knee arthroplasty (MTKA), who is inexperienced in RATKA, to make bone cuts and implant positions accurate to plan.

\section{Materials and Methods}

Six cadaveric specimens (12 knees) were prepared by a high-volume TKA surgeon, who had no prior clinical robotic-arm experience. For each cadaveric pair, a RATKA was prepared on the right leg and a MTKA was prepared on the left leg. Preoperatively, fiducial clusters were installed on each leg and a CT scan was obtained. Final bone cuts to plan were measured relative to the fiducials, representing final bone cut error (sum of bone registration error and bone cut error). Final component position to plan was also measured relative to the fiducials representing final bone cut error plus implantation and cementation error. Bone surfaces of the preoperative CTs were segmented to create three-dimensional plans with TKA component positional targets relative to the fiducials. An NDI Polaris optical tracking system and navigated probe were used to measure the positional differences of the final bone cuts and final component position when compared to the pre-operative plan. Medians and standard deviations for each final bone cut error and final component position error were compared between RATKA and MTKA for each planar bone cut and component position in the sagittal, coronal, and axial planes, for all six matched pairs. After the first three robotic-arm cases, the technique for bone registration was reviewed with the surgeon, and medians and standard deviations were compared between the last three matched pairs. Average RATKA/MTKA median or standard deviation ratios were also calculated for final bone cut and final component position to plan to compare overall accuracy and precision between RATKA and MTKA. It was assumed that the data followed a non-normal distribution, therefore, the median values were used to assess the central tendency of the data set. Accuracy to plan was defined by the median values of the absolute deviation from plan. Standard deviation was used to describe the precision to plan. For each measurement, 2-Variances testing was performed using alpha $=0.05$ for all six matched pairs, and not the reduced group with three matched pairs. P-value results indicate if a significant difference exists between the two operative methods [indicated by $(*)$, below]. Levene's test was used to assess the variances. V/V represents varus or valgus deviation, $\mathrm{F} / \mathrm{E}$ represents flexion or extension deviation, $\mathrm{I} / \mathrm{E}$ represents internal or external deviation, and $\mathrm{A} / \mathrm{P}$ represents anterior or posterior slope. 


\section{Results}

Measurements of medians show that RATKA bone cuts were more or as accurate to plan than the MTKA control, respectively for $11 / 12$ bone cut measurements: femoral anterior I/E $\left(0.9^{\circ}\right.$ vs. $\left.3.3^{\circ}\right)$, femoral anterior $\mathrm{F} / \mathrm{E}^{*}\left(0.4^{\circ}\right.$ vs. $\left.4.7^{\circ}\right)$, femoral anterior chamfer $\mathrm{V} / \mathrm{V}^{*}\left(0.5^{\circ} \mathrm{vs} .3^{\circ}\right)$, femoral anterior chamfer F/E $\left(0.3^{\circ}\right.$ vs. $\left.1.8^{\circ}\right)$, femoral distal V/V $\left(0.5^{\circ}\right.$ vs. $\left.2.6^{\circ}\right)$, femoral distal F/E $\left(0.8^{\circ}\right.$ vs. $\left.0.8^{\circ}\right)$, femoral posterior chamfer $\mathrm{V} / \mathrm{V}^{*}\left(1.1^{\circ}\right.$ vs. $\left.2.6^{\circ}\right)$, femoral posterior $\mathrm{I} / \mathrm{E}^{*}\left(1.0^{\circ}\right.$ vs. $\left.2.5^{\circ}\right)$, femoral posterior $\mathrm{F} / \mathrm{E}$ $\left(0.5^{\circ}\right.$ vs. $\left.2.3^{\circ}\right)$, tibial V/V* $\left(0.6^{\circ}\right.$ vs. $\left.1.2^{\circ}\right)$, and tibial A/P $\left(0.7^{\circ}\right.$ vs. $\left.0.9^{\circ}\right)$. The posterior chamfer F/E bone cut median was slightly higher for RATKA than MTKA $\left(0.9^{\circ}\right.$ vs. $\left.0.8^{\circ}\right)$.

Similarly, when comparing the standard deviations, RATKA bone cuts were more precise to plan than the MTKA control on all femoral bone cuts: femoral anterior I/E $\left(0.5^{\circ} \mathrm{vs} .1 .9^{\circ}\right)$, femoral anterior F/E* $\left(0.4^{\circ}\right.$ vs. $\left.2.3^{\circ}\right)$, femoral anterior chamfer V/V* $\left(0.1^{\circ}\right.$ vs. $\left.2.2^{\circ}\right)$, femoral anterior chamfer $\mathrm{F} / \mathrm{E}\left(0.2^{\circ}\right.$ vs. $\left.1.0^{\circ}\right)$, femoral distal V/V $\left(0.3^{\circ}\right.$ vs. $\left.1.6^{\circ}\right)$, femoral distal $\mathrm{F} / \mathrm{E}\left(0.5^{\circ} \mathrm{vs} .1 .1^{\circ}\right)$, femoral posterior chamfer $\mathrm{V} / \mathrm{V}^{*}\left(0.4^{\circ}\right.$ vs. $\left.2.0^{\circ}\right)$, femoral posterior chamfer $\mathrm{F} / \mathrm{E}\left(0.5^{\circ}\right.$ vs. $\left.1.6^{\circ}\right)$, femoral posterior $\mathrm{I} / \mathrm{E}^{*}\left(0.6^{\circ} \mathrm{vs}\right.$. $\left.1.6^{\circ}\right)$, and femoral posterior $\mathrm{F} / \mathrm{E}\left(0.6^{\circ} \mathrm{vs} .4 .0^{\circ}\right)$. The tibial $\mathrm{V} / \mathrm{V}^{*}$ bone cut was more precise for RATKA $\left(0.3^{\circ}\right.$ vs. $\left.0.7^{\circ}\right)$, but the tibial A/P cut was less precise for RATKA $\left(1.0^{\circ}\right.$ vs. $\left.0.3^{\circ}\right)$.

Robotic assisted total knee arthroplasty final component positions were as or more accurate to plan than the MTKA control for all measurements: femoral V/V* $\left(0.6^{\circ}\right.$ vs. $\left.3.2^{\circ}\right), \mathrm{F} / \mathrm{E}^{*}\left(0.6^{\circ}\right.$ vs. $\left.2.8^{\circ}\right), \mathrm{I} / \mathrm{E}$ $\left(0.8^{\circ}\right.$ vs. $\left.3.1^{\circ}\right)$, tibial V/V $\left(0.9^{\circ}\right.$ vs. $\left.0.9^{\circ}\right)$, tibial A/P $\left(1.1^{\circ}\right.$ vs. $\left.1^{\circ} .5^{\circ}\right)$. Additionally, RATKA final component positions were as or more precise to plan than MTKA for all femoral implant positions, as well as the tibial V/V implant positions: femoral V/V* $\left(0.3^{\circ} \mathrm{Vs} .1 .4^{\circ}\right), \mathrm{F} / \mathrm{E}^{*}\left(0.5^{\circ} \mathrm{Vs} .2 .1^{\circ}\right), \mathrm{I} / \mathrm{E}\left(0.5^{\circ} \mathrm{vs}\right.$. $\left.1.6^{\circ}\right)$, and tibial $\mathrm{V} / \mathrm{V}\left(0.4^{\circ}\right.$ vs. $\left.0.8^{\circ}\right)$, respectively.

A comparison of the standard deviations for the last three RATKA and MTKA matched pairs showed that RATKA was more precise to plan for all cuts and implant positions compared to MTKA, including tibial A/P bone cut $\left(0.2^{\circ}\right.$ vs. $0.5^{\circ}$ for RATKA and MTKA, respectively), and tibial implant A/P ( $0.4^{\circ}$ vs. $0.5^{\circ}$ for RATKA and MTKA, respectively). All data are presented in Figure 1, below.

The average RATKA/MTKA median ratio for all final bone cuts and final component positions showed RATKA was 4.2 and 3.2 times more accurate to plan that the MTKA control, respectively, for all six matched pairs. Similarly, the average RATKA/MTKA standard deviation ratio for all final bone cuts and final component positions showed RATKA was 5.0 and 3.1 times more precise to plan that the MTKA control, respectively, for all six matched pairs.

\section{Discussion and Conclusion}

Final bone cut and final component alignment can be critical for achieving a desired overall limb alignment and well-balanced knee. While surgical navigation helps improve implant planning, roboticarm technologies have emerged as a tool to help refine surgical execution [4]. Clinical evaluation of the variance in alignment in PKA procedures published by Lonner et al. [5], demonstrated lower root mean square error of the tibial slope $\left(1.9^{\circ}\right.$ vs. $\left.3.1^{\circ}\right)$ and varus/valgus $\left(0.2^{\circ} \pm 1.8^{\circ}\right.$ vs. $\left.2.7^{\circ} \pm 2.1^{\circ}\right)$ when using the Mako System compared to manual instrumentation. In this study of two consecutive series, 31 patients underwent Mako Partial Knee surgery while 27 patients underwent manual PKA. The variance using manual instrumentation was 2.6 times greater than the robotic-arm assisted bone preparation method in this study [5]. To the best of the authors' knowledge, this was the first study to assess TKA alignment using this specific robotic-arm system. Following a single cadaveric training with no previous RATKA experience, the surgeon's first six RATKA showed increased accuracy and precision to plan on all femoral bone cuts and implant positions, as well as the tibial V/V bone cut and implant position. Errors in tibial slope are attributed to the learning curve for tibial bone registration due to the surgeon's inexperience in RATKA and the process of bone registration, since the greatest deviation 
from plan for tibial slope cut and implant position occurred in the first two RATKA cases. Following the review of the tibial registration procedure, the accuracy for tibial slope improved, which is shown by comparing the median and standard deviation of the last three RATKA to 1) all six RATKA cases, and 2) the matched MTKA pairs. In general, RATKA demonstrated greater accuracy and precision of bone cuts and component placement to plan, compared to MTKA in this cadaveric study. In addition, the study demonstrated that RATKA has the potential to increase both the accuracy and precision of bone cuts and implant positioning to plan for an experienced manual surgeon who is new to RATKA. For further confirmation, RATKA accuracy of component placement should be investigated in a clinical setting.
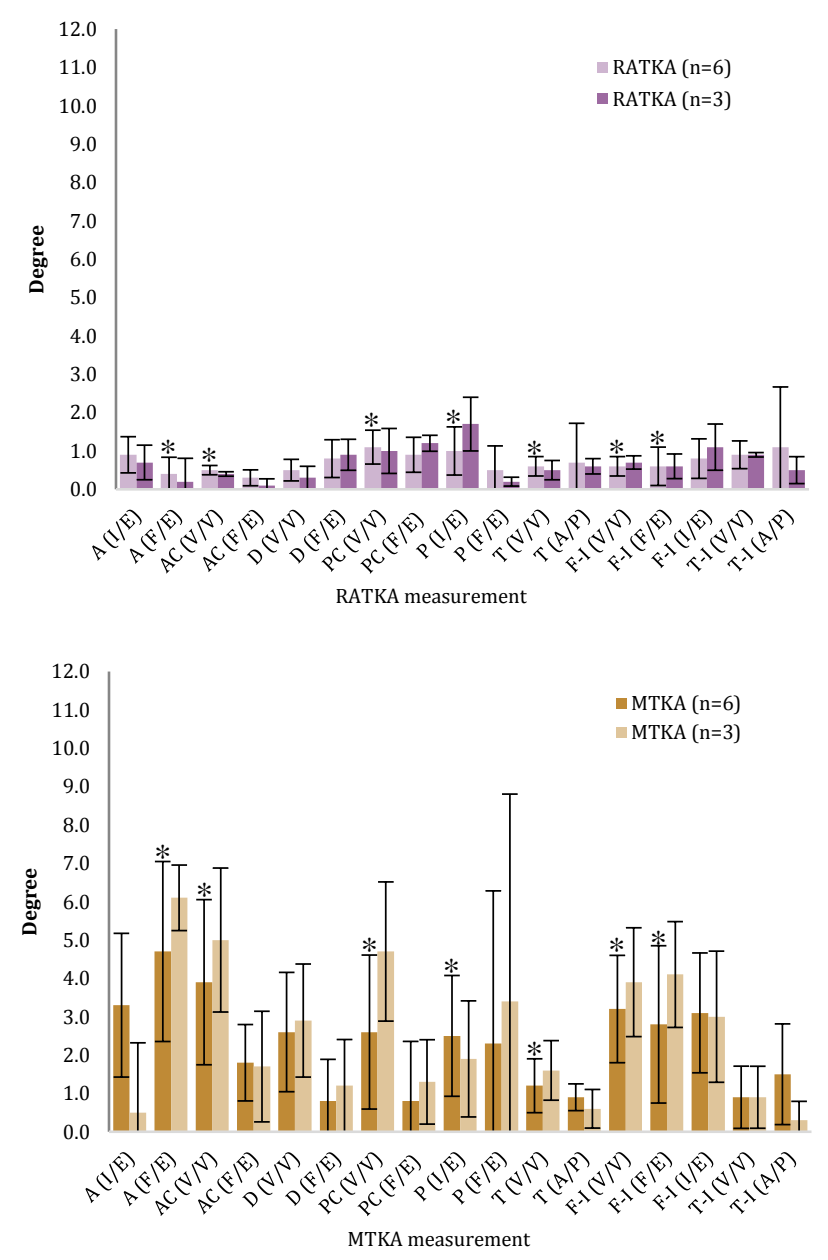

Figure 1: Comparison of RATKA and MTKA median cuts and implant position to plan for all 6 matched pairs $(n=6)$ and last 3 matched pairs $(n=3)$. For final bone cut to plan, on the femur, $A=$ Anterior, $\mathrm{AC}=$ Anterior Chamfer, $\mathrm{D}=$ Distal, $\mathrm{PC}=$ Posterior Chamfer, and $\mathrm{P}=$ Posterior, and on the tibia, $\mathrm{T}=$ Tibia. For final component position to plan, F-I $=$ Femoral Implant, T-I = Tibial Implant. V/V $=$ varus or valgus, $\mathrm{F} / \mathrm{E}=$ flexion or extension, $\mathrm{I} / \mathrm{E}=$ internal or external, and $\mathrm{A} / \mathrm{P}=$ anterior or posterior slope. Error bars represent standard deviation. *Indicates a significant difference between RATKA and MTKA for $\mathrm{n}=6$, with RATKA having less variance. 


\section{References}

[1] National Joint Registry (NJR) for England, Wales, Northern Ireland and the Isle of Man. 13th Annual Report. Available at:

http://www.njrreports.org.uk/Portals/0/PDFdownloads/NJR\%2013th\%20Annual\%20Report\%202016. pdf 2016. Accessed Dec. 10, 2016.

[2] Jauregui, JJ, Cherian, JJ, Pierce, TP, Beaver, WB, Issa, K, Mont, MA. Long-Term Survivorship and Clinical Outcomes Following Total Knee Arthroplasty. J Arthroplasty 30(12), pp. 2164-6, 2015.

[3] Mason JB, Fehring TK, Estok R, Banel D, Fahrbach K. Meta-analysis of alignment outcomes in computer-assisted total knee arthroplasty surgery. J Arthroplasty, 22(8), pp. 1097-1106, 2007.

[4] Carroll, KM, Pearle, AD. Computer Navigation and Robotics for Total Knee Arthroplasty. Am J Orthop. 44(6), pp. 251-252, 2015.

[5] Lonner JH, John TK, Conditt MA. Robotic-Arm Assisted UKA Improved Tibial Component Alignment: A Pilot Study Clin Orthop Relat Res. 468(1), pp.141-6, 2010.

\section{Disclosures}

Emily L. Hampp, Manoshi Bhowmik-Stoker, Laura Y. Scholl, and Jason K. Otto are Stryker employees. David J. Jacofsky and Michael A. Mont are consultants for Stryker. 